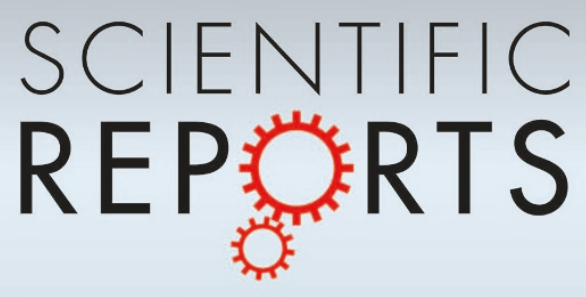

OPEN

SUBJECT AREAS:

OPTICAL MANIPULATION AND TWEEZERS

RHEOLOGY

MECHANICAL PROPERTIES

BIOPOLYMERS

Received

8 January 2013

Accepted

2 April 2013

Published

2 May 2013

Correspondence and requests for materials should be addressed to

H.R.-D. (halina@ physics.uq.edu.au)

\title{
Spatially-resolved rotational
} microrheology with an optically-trapped sphere

\author{
James S. Bennett' , Lachlan J. Gibson', Rory M. Kelly' , Emmanuel Brousse', Bastian Baudisch', \\ Daryl Preece', Timo A. Nieminen', Timothy Nicholson², Norman R. Heckenberg' \\ \& Halina Rubinsztein-Dunlop'
}

'The University of Queensland, School of Mathematics and Physics, Brisbane QLD 4072, Australia, ${ }^{2}$ The University of Queensland,
School of Chemical Engineering, Brisbane QLD 4072, Australia.

We have developed a microrheometer, based on optical tweezers, in which hydrodynamic coupling between the probe and fluid boundaries is dramatically reduced relative to existing microrheometers. Rotational

Brownian motion of a birefringent microsphere within an angular optical trap is observed by measuring the polarisation shifts of transmitted light. Data gathered in this manner, in the strongly viscoelastic fluid Celluvisc, quantitatively agree with the results of conventional (bulk) rheometry. Our technique will significantly reduce the smallest sample volumes which may be reliably probed, further extending the study of rare, difficult to obtain or highly nonhomogeneous fluids.

$T$ he field of microrheology, like the macroscopic study of flow and deformation, aims to characterise the manner in which fluids respond to applied stresses and/or strains. For soft materials this information is most commonly deduced by observing the motion of one or more microscopic probes embedded in the medium ${ }^{1}$. Microrheology has been applied in the study of a variety of physical systems, including polymer networks ${ }^{2-7}$, colloids $^{2,8-10}$ and fluids with mesoscopic nonhomogeneities ${ }^{4,11}$. These systems, collectively known as complex fluids, are said to be viscoelastic, as their response to deformation may exhibit characteristics of both elasticity and viscous dissipation. Complex fluids arise frequently in biology - examples include synovial fluid, tear films, lung surfactants and cerebrospinal fluid - and changes in their rheology have been linked to the development and diagnosis of diseased states ${ }^{12-14}$. Microrheology is therefore a powerful emerging tool for biophysics and medical diagnostics ${ }^{11,15}$.

The frequency-dependent behaviour of a viscoelastic fluid is described by the complex shear modulus, $G^{*}(\omega)$. The complex shear stress may be expressed as $\sigma(\omega)=G^{*}(\omega) \varepsilon(\omega)$, where $\varepsilon$ is the complex shear strain ${ }^{16}$. One can show that $G^{\prime}=\operatorname{Re}\left\{G^{*}\right\}$, the storage modulus, describes the amount of recoverable mechanical energy stored within the fluid. This is broadly similar to the interpretation of Young's modulus. Conversely, the loss modulus, $G^{\prime \prime}=\operatorname{Im}\left\{G^{*}\right\}$, reflects the momentum-dependent contribution of viscous damping.

The extreme nonhomogeneities and small volumes encountered in the study of many biological fluids ensure that the determination of $G^{*}$ by conventional rheometry is impractical. This necessitates the development of accurate microrheometers capable of reliably operating within ultra-small volume $(\sim \mathrm{pL})$ fluid samples. Such microrheometers must be relatively insensitive to the effects of hydrodynamic coupling between the probe particle $(s)^{10,17,18}$ and any fluid boundaries, potentially separated by only micrometres, as these interactions lead to a position-dependent decrease in the apparent mobility of the probe ${ }^{19,20}$. Overcoming these hurdles will allow continued miniaturisation of rheometry in lab-on-a-chip-style devices.

The influences of this coupling on the centre-of-mass and angular mobilities (in a viscous fluid) are described by Faxén's corrections ${ }^{20}$. The modification of the motion of a sphere near to a fluid boundary is specified as four coefficients; one each for the drag coefficients corresponding to rotations and translations parallel or perpendicular to the boundary. To leading order, the translational corrections for a particle of radius $a$ scale as $(a / s)^{1}$, whilst rotational corrections vary with $(a / s)^{3}$, with $s$ being the distance separating the probe's centre from the boundary $y^{20,21}$. As such, the rotational corrections do not become significant until $s \approx a$, allowing rotational microrheology to operate in more tightly confined geometries, or more heterogeneous fluids, than its translational counterpart ${ }^{20,21}$. As we will show below, this statement holds even relatively far from a boundary, despite 
the fact that rotational microrheology has a less favourable scaling of the uncertainty $\Delta G^{*}$ with $\Delta a$ than linear microrheology.

Previously, single-particle rotational optical microrheology has been restricted to measurements of viscosity ${ }^{19,22}$ or $\left|G^{*}\right|$, rather than $G^{\prime}$ and $G^{\prime \prime}$ individually. Herein we report the measurement of the full complex shear modulus, by a single-particle rotational microrheometer, across a large frequency range. We achieve excellent quantitative agreement between conventional rheological data and those gathered by monitoring the angular position fluctuations of a birefringent microsphere undergoing biased Brownian rotational motion in linearly polarised optical tweezers.

\section{Results}

The experimental apparatus is shown in Fig. 1. Our microrheometer exploits two laser sources; one forms a three-dimensional optical trap in an inverted microscope (Olympus, $1.3 \mathrm{NA}, 100 \times$ objective and matched condenser lenses) whilst another monitors the angular position of the trapped probe particle. The beams are coaxial and share a common focal spot at the position of the trap. The single optical trap is generated by highly-focused, linearly polarised infrared (IR) light (IPG Photonics YLR-5-1064-LP,CW, $1064 \mathrm{~nm}$ with stable output at $2.0 \mathrm{~W}$ ). An acousto-optic modulator (AOM, IntraAction DTD-274HA6, driven at $27 \mathrm{MHz}$ ) is aligned such that the first diffracted order is passed into the microscope, allowing fine control of the trapping power.

Vaterite $\left(\mathrm{CaCO}_{3}\right)$, our chosen probe material, forms highly birefringent $(\Delta n=0.1)$ polycrystalline microspherulites ${ }^{23}$. Despite non-trivial internal structure, the spheres have a well-formed principal optic axis, which allows the exchange of spin angular momentum with electromagnetic fields ${ }^{24}$. When held in linearly polarised optical tweezers these particles undergo biased diffusion about the stable mechanical equilibrium position, with the optic axis in the transverse plane and the azimuthal angle $\phi_{0}$ determined by the polarisation vector of the trapping beam.

Measurements of the angular position are achieved via polarisation tracking of a low-power ( $\sim 3 \mathrm{~mW}$ at focal plane) HeNe laser beam (JDS Uniphase 1137P, CW, $632.8 \mathrm{~nm}$ ) which is focused to a relatively broad focal spot. This tracking technique is related to those employed by Wood et al. and Knöner et al. ${ }^{25,26}$. Transmission through a vaterite microsphere deforms the initial circularly polarised beam into an elliptically polarised state, in which the long

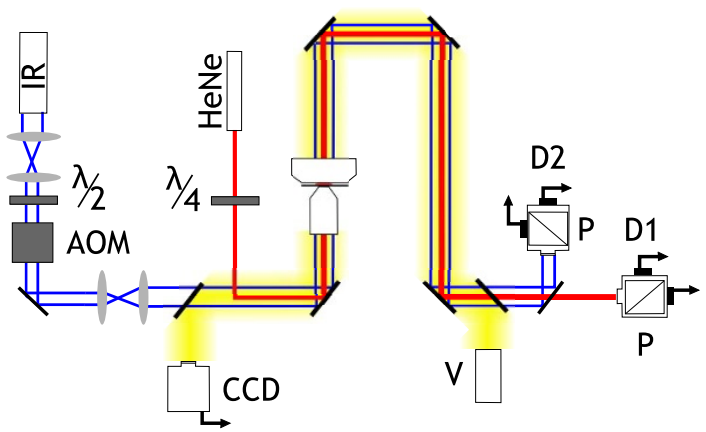

Figure 1 Experimental apparatus employed to monitor the directed rotational diffusion of a vaterite microsphere held in linearly polarised optical tweezers. Labels denote the infrared trapping laser (IR, $P \approx$ $20 \mathrm{~mW}$ at the trap, $\lambda=1064 \mathrm{~nm}$ ), circularly polarised HeNe tracking laser (HeNe, $P<3 \mathrm{~mW}, \lambda=632.8 \mathrm{~nm})$, half and quarter waveplates $(\lambda / 2$ or $\lambda / 4$ respectively), polarising beamsplitter $(\mathrm{P})$, acousto-optic modulator $(\mathrm{AOM})$, camera $(\mathrm{CCD})$, visible light source $(\mathrm{V})$ and polarisation-sensitive photodiode detectors (D1 and D2 for HeNe and IR beams respectively). The projection of the HeNe beam's polarisation ellipse onto a rectilinear basis allows the orientation of the vaterite probe particle to be recovered. The AOM allows control of the transmitted laser intensity. axis of the polarisation ellipse runs parallel to the optic axis of the probe. The resulting difference in the power carried by orthogonal (linearly polarised) beam components encodes $\phi$.

This information is recovered by passing the transmitted light through a polarising beam splitter cube and recording the difference in power arriving at each output port. Assuming the photodiode voltage is linear in the optical power, the recorded voltage difference varies sinusoidally with twice the azimuthal angle, $\Delta V-\langle\Delta V\rangle \propto \sin$ $2 \phi$, because of the probe's mirror symmetry about the optic axis. To recover absolute angles we observe the continuous rotation of the probe particle under circularly polarised IR light: once the extreme values of $\Delta V$ are known it is possible to map the data onto the interval $[-1,1]$ and solve for $\phi$ as a function of $\Delta V$.

Each individual probe is calibrated separately, requiring tens of seconds to one minute each, to ensure that variability of size and internal crystal structure ${ }^{23}$ within the population is properly accounted for. Note that continuous rotations induce restructuring of polymer networks present in strongly viscoelastic fluids, altering their apparent rheology or pushing them into the nonlinear regime ${ }^{1}$ : this potential source of data contamination has been avoided by collecting passive measurements of Brownian motion before calibrating.

The experimentalist concerned with small samples of nonhomogeneous fluids will find this system holds several advantages over existing microrheometers.

One of the simplest and most effective methods of measuring fluid properties is by monitoring the rectilinear motions of an ensemble of suspended probe particles. The well-developed field of optical multiprobe microrheology makes extensive use of dynamic light scattering $(\mathrm{DLS})^{27}$ and diffusing wave spectroscopy (DWS ${ }^{28}$, which generally extract data from the correlations of backscattered light. More specialised techniques, such as localised-coherence-volume DLS (capable of measurements localised to a volume of $\sim 100 \mu \mathrm{m}$ cubed $)^{29}$, are also in use. Additionally, the capacity for rapid multi-particle tracking afforded by modern CCD cameras has found applications in microrheology ${ }^{1,30}$.

Since the sample volume necessarily scales at least as rapidly as the number of probe particles (for any given technique) it is not possible for any of these techniques to approach the spatial resolution (the minimum distance over which a difference in $G^{*}$ may be detected) achieved by a single-probe microrheometer; they are ideally-suited to gathering large bodies of statistical data in near-homogeneous fluids. To increase the spatial resolution it is necessary to turn to few- and single-particle microrheometers.

Optical microrheometers based on translational motion of the probe particle(s) may monitor the Brownian motion of a trapped ${ }^{5,16}$ or untrapped ${ }^{31}$ probe, observe relaxation into an optical potential ${ }^{6,16}$ or continuously modulate the trap position ${ }^{5,11}$, amongst other possible schemes. All of these measurements rely on rectilinear motion, so are limited in spatial resolution by the strong dependence of the translational Faxén's corrections on the distance from a fluid boundary.

Our rotational microrheometer increases the achievable spatial resolution above those attained with the methods listed above through two mechanisms; firstly, the centre of the probe can be tightly constrained without degrading the signal-to-noise ratio, unlike translational measurements; and secondly, the rotational Faxén's corrections are small even within a radius of a fluid boundary.

Existing rotational microrheometers may also be categorised as ensemble or single-particle devices. In the former category are magnetic rheometers, which probe $G^{*}$ by examining the net magnetisation of a fluid interspersed with ferromagnetic probe beads - they are well-suited to measurement of low-frequency behaviour ${ }^{32,33}$, but are incapable of resolving $G^{*}$ at high frequencies $(\omega / 2 \pi>1 \mathrm{kHz})-$ and the rotational cousin of DLS, depolarised DLS ${ }^{34}$.

In the latter category are single-particle rotational microrheometers. Many of these are faced with a trade-off between angular 
sensitivity and ease of analysis; it is possible to optically track anisotropic and asymmetric objects, such as trapped wax micro disks ${ }^{3}$, but calculating their diffusive dynamics is highly nontrivial; conversely, the symmetry of spheres makes them difficult to track but simpler to analyse. We have circumvented this issue by employing a spherical particle with an optical anisotropy, allowing sensitive detection of its orientation with readily-available polarisers and photodetectors.

Finally, this Brownian microrheometer is able to acquire data far more rapidly than a similar instrument ${ }^{35}$ in which a birefringent sphere was sinusoidally driven with a well-defined frequency, because broad-band thermal forcing eliminates the necessity of scanning over each frequency of interest serially. A combination of these techniques could allow one to rapidly identify interesting features in $G^{*}$ by thermal effects, then perform high-resolution scans of those features with the active excitation method.

We adopt a generalised Langevin equation to model the stochastic evolution of the azimuthal angle of a spherical probe with moment of inertia $I$ and radius $a^{3}$. Without loss of generality we choose $\phi_{0}=0$.

$$
\ddot{I \phi}=\tau_{T H}(t)-\int_{-\infty}^{t} \zeta\left(t-t^{\prime}\right) \dot{\phi}\left(t^{\prime}\right) \mathrm{d} t^{\prime}-\chi \phi
$$

The angular position evolves under the effects of a Hookean optical restoring torque with effective stiffness $\chi$, the zero-mean thermal torque $\tau_{T H}$ and the fluid's hydrodynamic contributions, here represented by the convolution of the angular velocity with the microscopic memory function $\zeta(t)^{3}$.

The existence of a linear optical restoring torque is confirmed by experiments, as clearly shown in Fig. 2. The distribution of occupied angular positions is in accordance with a thermal ensemble within a harmonic potential. Therefore the angular trap stiffness may be confidently determined by way of the equipartition theorem. Note that this calculation is independent of the fluid's rheological properties.

$$
\chi=\frac{k_{B} T}{\left\langle\phi^{2}\right\rangle}
$$

Here brackets $\langle\cdots\rangle$ indicate a measurement averaged over all $t$; experimentally, the measurement time must be sufficiently long that the mean-squared displacement approaches a constant value.

The normalised angular position autocorrelation function (NAPAF), defined by Eqn. (3), contains information concerning the distribution of $\phi(t+\tau)$ conditioned on a measurement of $\phi(t)$. As such, it encapsulates the dynamical behaviour of the probe.

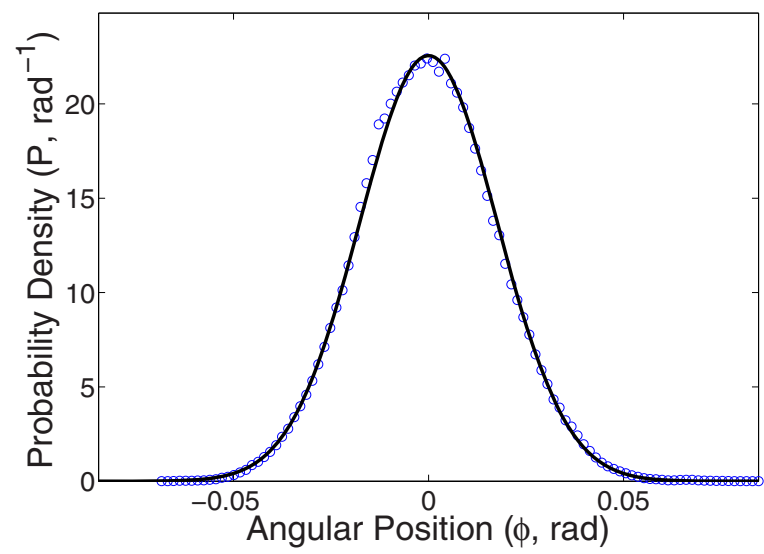

Figure $2 \mid$ The distribution of angular positions is consistent with a thermally-populated effective harmonic optical potential. An experimental histogram of vaterite orientations (circles), collected in 25\% Celluvisc, is shown against a normalised Gaussian (solid line) of the form $p_{0} \exp \left[-\phi^{2} / 2\left\langle\phi^{2}\right\rangle\right]$. The close agreement indicates that the optical restoring torque is linear in $\phi$ within the region sampled by the particle.

$$
A(\tau)=\frac{\langle\phi(t) \phi(t+\tau)\rangle}{\left\langle\phi(t)^{2}\right\rangle},
$$

By analogy with Preece et al. ${ }^{16}$, we apply the generalised StokesEinstein relation of Cheng and Mason ${ }^{3}$ to Eqn. (1) to find

$$
G^{*}(\omega)=\frac{1}{8 \pi a^{3}}\left[I \omega^{2}-\frac{\chi \omega \tilde{A}(\omega)}{i+\omega \tilde{A}(\omega)}\right] .
$$

It is important to note that $\tilde{A}$ is representative of the unilateral Fourier transform ${ }^{1}$ of $A$, defined as

$$
\tilde{A}(\omega)=\int_{0}^{\infty} A(\tau) e^{-i \omega \tau} \mathrm{d} \tau .
$$

Numerical evaluation of Eqn. (5) is performed using an approximation closely related to that of Evans et al. ${ }^{36}$. This method treats the experimental data as a piecewise linear function, interpolating between adjacent points. Times $\tau$ beyond the experimentally probed region are integrated over by extrapolating $A(\tau)$ with an appropriate function.

In order to choose said function we note that, for a simple viscous fluid in the low Reynolds number (R) regime ${ }^{16}$, Eqn. (3) reduces to

$$
A(\tau)=e^{-\alpha \tau}=\exp \left[-\frac{\chi \tau}{8 \pi a^{3} \eta}\right] .
$$

The majority of materials will respond in an essentially viscous manner if deformed over timescales much greater than any characteristic relaxation times of their substructures, allowing us to apply Eqn. (6) as an approximation to the long-time behaviour of the NAPAF measured in complex fluids.

Given a uniformly-sampled set of $N$ points $A_{j}$ spaced by $\Delta t$ in the domain $\tau \in\left[0, \tau_{N}\right]$, we extrapolate using $A_{e x}=A_{N} e^{-\alpha} \mathrm{eff}^{\left(\tau-\tau_{N}\right)}$ to find

$$
\begin{gathered}
\tilde{A}(\omega)=\frac{\left(1-e^{i \omega \Delta t}\right) \sum_{j=1}^{N-1}\left(A_{j+1}-A_{j}\right) e^{-i \omega j \Delta t}+}{} \frac{A_{1}-A_{N} e^{-i \omega \tau_{N}}}{i \omega}+\frac{A_{N} e^{-i \omega \tau_{N}}}{i \omega+\alpha_{\mathrm{eff}}} .
\end{gathered}
$$

The extrapolating function introduces only a single fitting parameter, the effective decay constant $\alpha_{\text {eff. }}$

Much of the theory presented above is predicated on the existence of low- $\mathscr{R}$ flows. We confirm that we are probing low- $\mathscr{R}$ dynamics by comparing the experimentally determined NAPAF (water) with its corresponding theoretical form in this regime, Eqn. (6). As seen in Fig. 3, the agreement between these is excellent. This indicates that Eqn. (4) may be simplified by neglecting the inertial terms $\left(\propto I \omega^{2}\right)^{25}$.

We benchmark our method against conventional techniques by examining four aqueous dilutions of a common dry eye treatment, Celluvisc (Allergan). This fluid, a carboxymethylcellulose (CMC) sodium solution $\left(10 \mathrm{mg} \mathrm{mL}^{-1}\right)$, is designed to mimic the rheological properties of the fluid film coating the cornea. CMC solutions are quite rheologically stable, with unpreserved samples aging over a number of weeks ${ }^{37}$.

Microrheological measurements were conducted in dilutions of 100, 50, 25 and 0 percent Celluvisc by weight, with trapping powers of approximately $20-60 \mathrm{~mW}$. Rheological properties were found to be essentially independent of the trapping power over this range, as noted in previous experiments ${ }^{22}$. Each measurement was carried out over three to five minutes.

The bulk response of each fluid was probed with a parallel-plate strain controlled rheometer (ARES, Rheometric Scientific). With time-temperature superposition at approximately 8,25 and $40^{\circ} \mathrm{C}$ we were able to achieve measurements of $G^{*}$ across the range $\omega \in$ $[1,200] \mathrm{rad} \mathrm{s}^{-1}$ without entering the operating regime in which the inertia of the instrument significantly affects data. Although these 


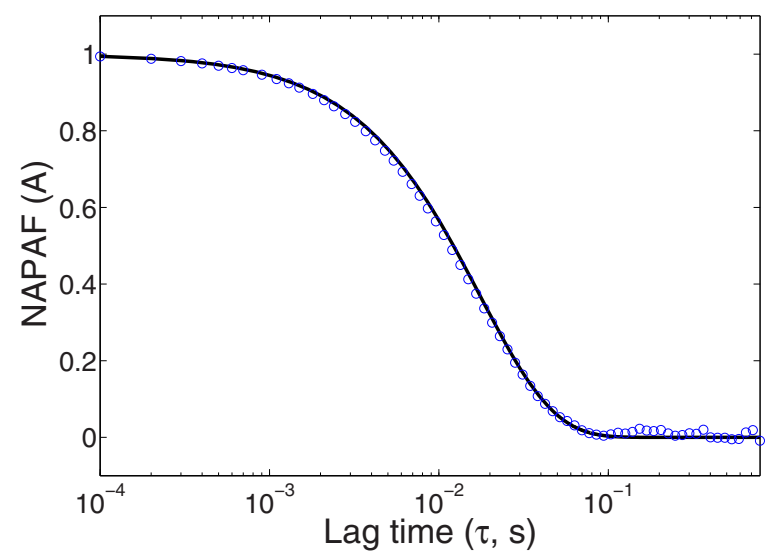

Figure 3 Experimental autocorrelation functions collected in samples of water are in close agreement with their expected forms. A decaying exponential function (solid line) is fitted to the experimental data (circles), with a single free parameter. We find the viscosity to be $\eta=(0.97 \pm$ 0.16) $\mathrm{mPa} \cdot \mathrm{s}$, consistent with the known viscosity near to room temperature. The raw data were collected in a single run of five minutes and are presented with no averaging or filtering.

frequencies are comparatively low, the region of overlap with our tweezers-based method is sufficient for critical analysis.

\section{Discussion}

In optimal conditions measurements of $G^{*}$ were repeatable and consistent. The largest sources of uncertainty in the moduli stem from the low-frequency motion of the apparatus, which pollutes the NAPAF, and an inability to precisely determine the probe radius. The former was able to be partially mitigated by high-pass filtering the raw time-series prior to analysis (passing $\omega>1 \mathrm{rad} \mathrm{s}^{-1}$ ). It may be possible to further suppress low-frequency noise by employing two (mutually incoherent) trapping beams, allowing high (rectilinear) trap stiffnesses whilst maintaining a weak angular trap.

The latter, which introduces a particle radius uncertainty, contributes a goodly portion of the final experimental uncertainty in $G^{*}$. With our estimated uncertainty of $\Delta a \approx 0.1 a$, the relative uncertainty in $1 / a^{3}$ is $\approx 30 \%$; this is smaller than the systematic error associated with translational wall effects if the centre of the particle is within five radii of the boundary. This increases to $s \approx 9 a$ if the uncertainty is reduced to $\Delta a \approx 0.05 a$, illustrating that rotational microrheometers

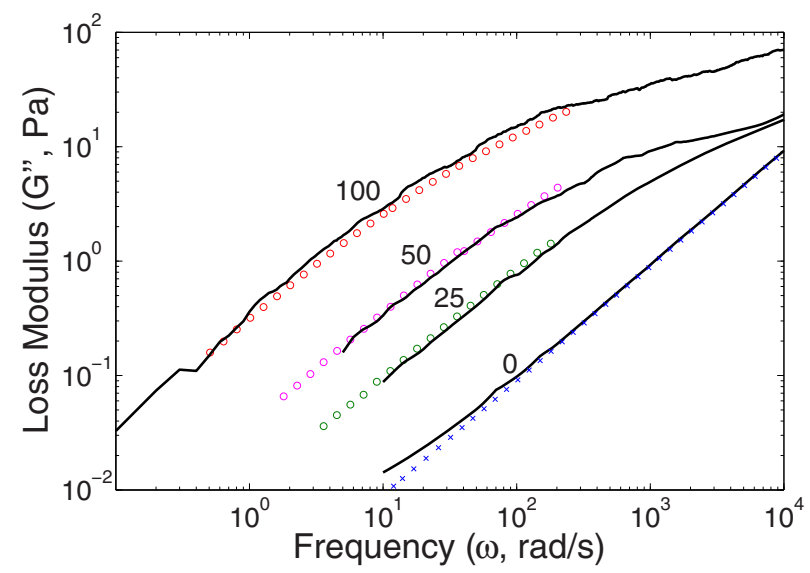

Figure $4 \mid$ A comparison of the loss modulus of aqueous dilutions of Celluvisc $(100,50,25$ and $0 \%$ by weight) measured by bulk (circles) and microrheometric (lines) techniques. The (theoretical) loss modulus of water (crosses) is of the form $G^{\prime \prime}=\omega \eta$. All data show excellent agreement over a frequency range in excess of a decade.

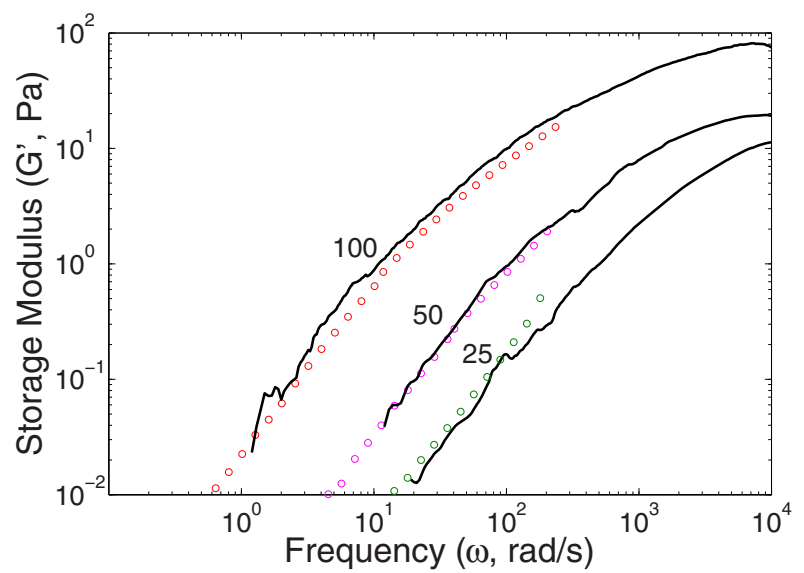

Figure $5 \mid$ The storage modulus of aqueous dilutions of Celluvisc (100, 50 and $25 \%$ by weight), measured by conventional (circles) and microrheological (lines) techniques. Water $\left(G^{\prime}=0\right)$ is omitted for clarity. Apart from small deviations (discussed in text) these data closely agree.

are capable of outperforming their translational counterparts even relatively far from a wall. We also note that if the same probe particle is used to make comparative measurements, which are of immense interest to the biophysicist, the uncertainty in the radius does not affect the results.

Data were prepared for presentation by linearly interpolating each of $G^{\prime}$ and $G^{\prime \prime}$ across four independent measurements, averaging these and selecting a logarithmically-spaced set of representative points. We do not display $G^{*}\left(\omega>10^{4} \mathrm{~Hz}\right)$ due to the presence of highfrequency artefacts caused by our finite sampling rate $\mathrm{rat}^{31,38}$.

Fig. 4 and Fig. 5 compare our microrheological measurements with conventional rheometry. The data compare favourably over the frequency range in which they overlap, spanning at least one decade. Slight deviations are evident in $G^{\prime}$ measured in 25 percent solution, at frequencies exceeding $10^{2} \mathrm{rad} \mathrm{s}^{-1}$. Although it is difficult to disentangle possible contributing factors, we believe that this difference may be due to depletion interactions ${ }^{2}$ or the breakdown of the continuum approximation ${ }^{3}$.

We note that the high-frequency behaviour of $G^{\prime \prime}$ is consistent with shear thinning, also observed in methylcellulose solutions ${ }^{5}$.

Our novel microrheological scheme has proven to reliably measure properties of complex fluids in small fluid samples. By analysing the rotational motion of a single probe particle we have reduced the minimum volume of fluid required for a characterisation of its viscoelastic properties. Furthermore, our analysis relies on only a few key assumptions, particularly those relevant to the generalised Stokes-Einstein relation ${ }^{1}$, so should be robust within the realms of their validity.

This technique will allow more detailed analysis of biological fluids, potentially for rapid and reliable diagnostics, and will increase the range of (bio)physical studies able to be carried out inside living cells ${ }^{39,40}$ and in chip-based devices.

\section{Methods}

Apparatus. The arrangement of optical devices is depicted in Fig. 1. The IR laser (IPG Photonics YLR-5-1064-LP, CW, $1064 \mathrm{~nm}$ with stable output at $2.0 \mathrm{~W}, P \approx 10 \mathrm{~mW}$ at the trap) is focused through an inverted high-NA microscope (Olympus, 1.3 NA, $100 \times$ objective and matched condenser lenses) to form the optical trap. A HeNe laser (JDS Uniphase 1137P, CW, $632.8 \mathrm{~nm}, P<3 \mathrm{~mW}$ at focal plane) and plate are used for polarisation tracking. The AOMs (IntraAction DTD-274HA6, driven at $27 \mathrm{MHz}$ with variable amplitude) allows the trapping power to be controlled.

Fluids. Vaterite spheres were suspended in samples of Celluvisc (Allergan), a $10 \mathrm{~g} / \mathrm{L}$ aqueous solution of CMC, after removal of the mother liquor through three repeated cycles of centrifugation, re-suspension in ethanol and sonication.

Angle calibration. The transmitted HeNe light was passed through a polarising beam splitter cube and the resulting powers of both perpendicular beams measured. The 
voltage difference generated $(\Delta V)$ relates sinusoidally to the azimuthal angle of the vaterite probe by $\Delta V \propto \sin 2 \phi$. Introducing a quarter wave plate into the IR path causes the vaterite to spin. Recording the range of $\Delta V$ allows an exact relationship between $\Delta V$ and $\phi$ to be found.

Large scale rheometry. A parallel-plate strain controlled rheometer (ARES, Rheometric Scientific) with sample temperatures of approximately 8,25 and $40^{\circ} \mathrm{C}$ achieved measurements of $G^{*}$ across the frequency range 1-200 rad/s by standard time-temperature superposition.

1. Squires, T. M. \& Mason, T. G. Fluid mechanics of microrheology. Annual Review of Fluid Mechanics 21, 413-438 (2009).

2. Starrs, L. \& Bartlett, P. Colloidal dynamics in polymer solutions: Optical twopoint microrheology measurements. Faraday Discussions 123, 323-334 (2003)

3. Cheng, Z. \& Mason, T. G. Rotational diffusion microrheology. Physical Review Letters 90, 018304 (2003).

4. Mizuno, D., Head, D. A., Mackintosh, F. C. \& Schmidt, C. F. Active and passive microrheology in equilibrium and nonequilibrium systems. Macromolecules 41, 7194-7202 (2008)

5. Brau, R. R., Ferrer, J. M., Lee, H., Castro, C. E. \& Tam, B. K. Passive and active microrheology with optical tweezers. Journal of Optics A 9, 102-112 (2007).

6. Tassieri, M. et al. Measuring storage and loss moduli using optical tweezers: broadband microrheology. Physical Review E 81, 026308 (2010).

7. Gibson, G. M., Leach, J., Keen, S., Wright, A. J. \& Padgett, M. J. Measuring the accuracy of particle position and force in optical tweezers using high-speed video microscopy. Optics Express 16, 405-412 (2008).

8. Otto, O. et al. High-speed video-based tracking of optically trapped colloids. Journal of Optics 13, 044011 (2011).

9. Wilson, L. G., Harrison, A. W., Poon, W. C. K. \& Puertas, A. M. Microrheology and the fluctuation theorem in dense colloids. EPL 93, 58007 (2011).

10. Wang, G. M., Prabhakar, R., Gao, Y. X. \& Sevick, E. M. Micro-rheology near fluid interfaces. Journal of Optics 13, 044009 (2011).

11. Wilson, L. G. \& Poon, W. C. K. Small-world rheology: an introduction to probebased active microrheology. Physical Chemistry Chemical Physics 13, 10617-10630 (2011)

12. Thurston, G. B. \& Greiling, H. Viscoelastic properties of pathological synovial fluids for a wide range of oscillatory shear rates and frequencies. Rheologica Acta 17, 433-445 (1978).

13. Rantamäki, A. H., Telenius, J., Koivuniemi, A., Vattulainen, I. \& Holopainen, J. M. Lessons from the biophysics of interfaces: Lung surfactant and tear fluid. Progress in Retinal and Eye Research 30, 204-215 (2011).

14. Kazakov, V. N. et al. Dilation rheology as medical diagnostics of human biological liquids. Colloids and Surfaces A: Physicochemical and Engineering Aspects 391 190-194 (2011).

15. Weihs, D., Mason, T. G. \& Teitell, M. A. Bio-microrheology: A frontier in microrheology. Biophysical Journal 91, 4296-4305 (2006).

16. Preece, D. et al. Optical tweezers: wideband microrheology. Journal of Optics 13, $044022(2011)$

17. di Leonardo, R. et al. Eigenmodes of a hydrodynamically coupled micron-size multiple-particle ring. Physical Review E 76, 061402 (2007).

18. Hough, L. A. \& Ou-Yang, H. D. Correlated motions of two hydrodynamically coupled particles confined in separate quadratic potential wells. Physical Review $E$ 65, 021906 (2002)

19. Parkin, S. J., Knöner, G., Nieminen, T. A., Heckenberg, N. R. \& RubinszteinDunlop, H. Picoliter viscometry using optically rotated particles. Physical Review E 76, 041507 (2007)

20. Leach, J. et al. Comparison of Faxén's correction for a microsphere translating or rotating near a surface. Physical Review E 79, 026301 (2009).

21. Berg-Sørensen, K. \& Flyvbjerg, H. Power spectrum analysis for optical tweezers. Review of Scientific Instruments 75, 594-612 (2004).

22. Bishop, A. I., Nieminen, T. A., Heckenberg, N. R. \& Rubinsztein-Dunlop, H. Optical microrheology using rotating laser-trapped particles. Physical Review Letters 92, 14-17 (2004)

23. Vogel, R. et al. Synthesis and surface modification of birefringent vaterite microspheres. Langmuir 25, 11672-11679 (2009).

24. Funk, M. et al. Constant power optical tweezers with controllable torque. Optics Letters 34, 139-141 (2009).
25. Wood, T. A., Roberts, G. S., Eaimkhong, S. \& Bartlett, P. Characterization of microparticles with driven optical tweezers. Faraday Discussions 137, 319-333 (2008).

26. Knöner, G., Parkin, S., Heckenberg, N. R. \& Rubinsztein-Dunlop, H. Characterization of optically driven fluid stress fields with optical tweezers. Physical Review E 72, 031507 (2005).

27. Dasgupta, B. R., Tee, S.-y., Crocker, J. C., Frisken, B. J. \& Weitz, D. A. Microrheology of polyethylene oxide using diffusing wave spectroscopy and single scattering. Physical Review E 65, 051505 (2002).

28. Weitz, D. A., Zhu, J. X., Durian, D. J., Gang, H. \& Pine, D. J. Diffusing-wave spectroscopy: The technique and some applications. Physica Scripta T49, 610-62 (1993).

29. Popescu, G., Dogariu, A. \& Rajagopalan, R. Spatially resolved microrheology using localized coherence volumes. Physical Review E 65, 041504 (2002).

30. Kurniawan, N. A., Lim, C. T. \& Rajagopalan, R. Image correlation spectroscopy as a tool for microrheology of soft materials. Soft Matter 6, 3499-3505 (2010).

31. Pesce, G., Rusciano, G. \& Sasso, A. Blinking Optical Tweezers for microrheology measurements of weak elasticity complex fluids. Optics Express 18, 2116-2126 (2010).

32. Besseris, G. J., Miller, I. F. \& Yeates, D. B. Rotational magnetic particle microrheometry: The Newtonian case. Journal of Rheology 43, 591-608 (1999).

33. Wilhelm, C., Browaeys, J., Ponton, A. \& Bacri, J.-C. Rotational magnetic particles microrheology: The Maxwellian case. Physical Review E 67, 011504 (2003).

34. Andablo-Reyes, E., Diaz-Leyva, P. \& Luis Arauz-Lara, J. Microrheology from rotational diffusion of colloidal particles. Physical Review Letters 94, 106001 (2005).

35. Funk, M., Parkin, S. J., Nieminen, T. A., Heckenberg, N. R. \& Rubinsztein-Dunlop, $\mathrm{H}$. Vaterite twist: microrheology with AOM controlled optical tweezers. Proceedings of SPIE 7227, 72270D (2009).

36. Evans, R., Tassieri, M., Auhl, D. \& Waigh, T. A. Direct conversion of rheological compliance measurements into storage and loss moduli. Physical Review E 80, 1-4 (2009).

37. Naik, S. C., Lee, J. C. \& Richardson, J. F. The rheology and aging characteristics of sodium carboxymethylcellulose solutions. The Canadian Journal of Chemical Engineering 55, 90-92 (1977).

38. Fusco, S. et al. High frequency viscoelastic behaviour of low molecular weight hyaluronic acid water solutions. Biorheology 44, 403-418 (2007).

39. Sacconi, L., Tolić-Nørrelykke, I. M., Stringari, C., Antolini, R. \& Pavone, F. S. Optical micromanipulations inside yeast cells. Applied Optics 44, 2001-2007 (2005).

40. Oddershede, L., Flyvbjerg, H. \& Berg-Sørensen, K. Single-molecule experiment with optical tweezers: improved analysis of the diffusion of the $\lambda$-receptor in $\mathrm{E}$ coli's outer membrane. Journal of Condensed Matter Physics 15, S1737 (2003).

\section{Acknowledgements}

The authors gratefully acknowledge Mr Jim Johnson and Dr Jennifer Steen for supplying the viscoelastic materials examined in this work. We also thank Dr Alex Stilgoe for assistance with the experimental apparatus and Dr Manlio Tassieri for useful conversations. This work was supported by an Australian Council Research Discovery Grant, DP110103015.

\section{Author contributions}

J.S.B., L.J.G. and R.M.K. performed data collection and analysis. E.B., B.B., J.S.B., D.P. and L.J.G. constructed the microrheometer. T.A.N. provided theoretical support. T.N. collected and analysed macrorheological data. This manuscript was developed by J.S.B. in collaboration with L.J.G., D.P., T.A.N. and H.R.-D. The technique was conceived by T.A.N., N.R.H. and H.R.-D.

\section{Additional information}

Competing financial interests: The authors declare no competing financial interests.

License: This work is licensed under a Creative Commons

Attribution-NonCommercial-NoDerivs 3.0 Unported License. To view a copy of this license, visit http://creativecommons.org/licenses/by-nc-nd/3.0/

How to cite this article: Bennett, J.S. et al. Spatially-resolved rotational microrheology with an optically-trapped sphere. Sci. Rep. 3, 1759; DOI:10.1038/srep01759 (2013). 